\title{
Determination of Benzodiazepines in Clinical Serum Samples: Comparative Evaluation of REMEDi System, aca Analyzer, and Conventional HPLC Performance
}

\author{
Tsui-Ling Chang, ${ }^{1 *}$ Kuan-Wen Chen, ${ }^{2}$ Yih-Dar Lee, ${ }^{3}$ and Kang Fan ${ }^{4}$ \\ ${ }^{1}$ Laboratory of Toxicology, Department of Pathology, National Cheng Kung University Hospital, \\ Tainan, Taiwan \\ ${ }^{2}$ Department of Emergency, National Cheng Kung University Hospital, Tainan, Taiwan \\ ${ }^{3}$ Department of Psychiatry, National Cheng Kung University Hospital, Tainan, Taiwan \\ ${ }^{4}$ Department of Pathology \& Laboratory Medicine, John L. McClellan Memorial VA Hospital, \\ Little Rock, Arkansas
}

\begin{abstract}
Emergency toxicology or drug screening in clinical settings requires rapid qualitative and quantitative analysis with acceptable levels of sensitivity and specificity. The aim of this study was to comparatively evaluate the performance of the multi-column HPLCbased REMEDi drug profiling system (BioRad), the aca analyzer (Du Pont), and the bench standard conventional HPLC method in the identification of 12 clinically important benzodiazepines. In this study, the presence of benzodiazepines in 133 patients' serum samples were qualitatively and comparatively analyzed by these three procedures. Among these methods, 120 of 133 samples were identified as benzodiazepine-positive by conventional HPLC (90\%); 127 by aca analyzer (95\%); and 84 by REMEDi $(63 \%)$. Detection sensitivity of REMEDi for most of the benzodiazepines was found satisfactory when concentrations were greater than 1.0 $\mu \mathrm{g} / \mathrm{mL}$. When benzodiazepine concentrations were in the ranges of $0.3-1.0 \mu \mathrm{g} / \mathrm{mL}$, detection sensitivity became varied among the benzodiazepine family of drugs by REMEDi method. REMEDi procedure should not be considered as the method of choice

for detection of benzodiazepines if expected concentration levels are below $0.3 \mu \mathrm{g} / \mathrm{mL}$, with the exception of bromazepam. Conventional HPLC displayed the highest sensitivity and specificity for the detection of benzodiazepines. In our studies, 36 REMEDi-negative samples were positive by HPLC, although in 16 of the 36 REMEDi negative samples (13.3\%), the presence of benzodiazepines was detected but only listed as candidates without positive identification of the individual compounds by REMEDi. In our series, however, there were no false positives by the REMEDi method whereas aca procedure showed 13 false positive results (9\%) and 6 cases of false negative (4\%).

Our data indicate that the REMEDi procedure can be used on serum samples for rapid qualitative screening of clinically important high levels of benzodiazepines with high specificity. However, due to the relatively low sensitivity of REMEDi in samples with low benzodiazepine levels and relatively low specificity by aca, all samples should be further confirmed by conventional HPLC procedure. J. Clin. Lab. Anal. 13:106-111, 1999. (c)1999 Wiley-Liss, Inc.
\end{abstract}

Key words: benzodiazepines; REMEDi; serum; HPLC; aca analyzer

\section{INTRODUCTION}

Detection of benzodiazepines, a class of frequently prescribed drugs and candidates for drug abuse, is an important component of emergency toxicology and drug abuse testing. In a clinical setting, an ideal analytical method for the detection of benzodiazepines should be rapid, sensitive, and specific, as well as have the ability to detect many different compounds of the same class, preferably in the same procedure and in the same sample (1). In our hospital, we routinely used immunoassay (aca analyzer) to screen for the presence of benzodiazepines in serum samples (2). Although immunoassay is able to rapidly provide qualitative information on certain groups of structurally-related compounds, it suffers from two disadvantages. First, immunoassay does not pro-

Grant sponsor: The National Cheng Kung University Hospital Research Council; Grant number: NCKUH-86-063.

*Correspondence to: Tsui-Ling Chang, Laboratory of Toxicology, Department of Pathology, National Cheng Kung University Hospital, 138 ShengLi Road, Tainan, Taiwan, R.O.C. E-mail: tsuiling @ mail.ncku.edu.tw

Received 15 September 1998; Accepted 29 January 1999. 
vide a precise identification of the individual benzodiazepines or their metabolites. In clinical toxicology settings, it is important to identify different drugs of the same class since their biological effects may differ widely. Second, unless the suspect drug or metabolite is known and an antibody is available, an immunoassay will not provide information for that specific compound. In clinical situations, we often encounter serum samples with a significant level of benzodiazepine, but the sample may also contain other clinically significant medications or illicit drugs that are not detectable by single-purposed immunoassay.

In the qualitative analysis of clinically important medicinal compounds, many chromatographic methods are routinely used to identify individual drugs and their metabolites. Routine procedures include thin layer chromatography (TLC), gas chromatography (GC), and analyte-optimized high performance liquid chromatography (HPLC).

To evaluate whether REMEDi (Bio-Rad, Hercules, CA), an HPLC-based broad-spectrum drug profiling system, could be used to reliably and rapidly identify 12 benzodiazepine family members (alprazolam, bromazepam, clonazepam, chlordiazepoxide, diazepam, estazolam, flunitrazepam, flurazepam, lorazepam, nitrazepam, triazolam, and midazolam) in clinical serum samples and simultaneously detect any polypharmacy in these samples, we evaluated the performance of REMEDi procedure and compared the results with data obtained from aca and standard HPLC methods. Although the REMEDi procedure is primarily designed to promptly identify basic and neutral drugs and their metabolites in urine, it can also be modified for the identification of benzodiazepines and barbiturates in serum samples (3-9). Data to be presented suggest that REMEDi is a viable procedure for rapid screening of serum sample for the detection of clinically significant levels of benzodiazepines and compared favorably to HPLC in specificity but suffers from low sensitivity whereas ACAprocedure provides high sensitivity but lowest specificity among these three procedures.

\section{MATERIALS AND METHODS}

\section{Patient Samples}

Clinical serum specimens were obtained from 133 patients who were suspected of illicit benzodiazepines usage. Samples were collected from the emergency and psychiatric departments of our hospital between January 1993 and July 1996. The samples were initially screened for the presence of benzodiazepine-like immunoactivity by aca analyzer and the results were further confirmed by conventional HPLC. In this study, these 133 samples were used for the evaluation of REMEDi performance.

\section{Immunoassay (aca Analyzer)}

The Du Pont (Wilmington, DE) aca discrete clinical analyzer serum benzodiazepines screen (S BNZ) method was performed according to the instructions of the manufacturer. The calibrator is diazepam. The result of an S BNZ analysis is given in QUAL unit, a relative unit. For standards containing pure diazepam, relative QUAL units are equivalent to the drug concentration in $\mu \mathrm{g} / \mathrm{mL}$. For other benzodiazepines, QUAL unit is not equivalent to drug concentration. If the QUAL unit was converted to $\mu \mathrm{g} / \mathrm{mL}$ by extrapolating from standard concentration curve, the LOD of some benzodiazepines, in our hands, as follows: chlordiazepoxide (5.0), clonazepam (2.0), diazepam (0.3), flurazepam (2.0), lorazepam (3.0), nitrazepam (2.0) (all expressed in $\mu \mathrm{g} / \mathrm{mL}$ ). The LOD for bromazepam, estazolam, alprazolam, midazolam and flunitrazepam were not determined by aca method. Triazolam could cross-react with other benzodiazepines hence the LOD for triazolam also was not determined. Although the standard unit for aca is expressed in QUAL unit, in this report, we converted the data to $\langle\mu \mathrm{g} / \mathrm{mL}$ whenever possible. Hemolysis (hemoglobin $<418 \mathrm{mg} / \mathrm{dL}$ ), icterus (total bilirubin $<10 \mathrm{mg} / \mathrm{dL}$ ), and lipidemia (triglyceride $<510 \mathrm{mg} / \mathrm{dL}$ ) do not interfere with the S BNZ method.

\section{HPLC}

Two (2) $\mathrm{mL}$ of serum sample was transferred to a screwcapped tube, and $20 \mu \mathrm{L}$ of internal standard solution (acetaminophen, $5 \mathrm{mg} / \mathrm{mL}$ in methanol) was added. The mixture was extracted with $5 \mathrm{~mL}$ of heptane/ethyl acetate/dichlormethane $(50: 45: 55, \mathrm{v} / \mathrm{v})$. The organic layer was transferred to a separate vial and evaporated to dryness. The residue was dissolved in $100 \mu \mathrm{L}$ methanol and $10 \mu \mathrm{L}$ was injected onto an ODS Hyperil $5 \mu \mathrm{m}, 200 \times 4.6 \mathrm{~mm}$ column. An HP 1050 series HPLC system (Hewlett Packard, Palo Alto, CA) was used. The mobile phase was (Phosphate buffer:acetonitrile $=70: 30$ ), wavelength 210 $\mathrm{nm}$ and the flow rate was $1.0 \mathrm{~mL} / \mathrm{min}$. The total separation time was $50 \mathrm{~min}$. The LOD for the 12 benzodiazepines tested by HPLC procedure was uniformly $0.2 \mu \mathrm{g} / \mathrm{mL}$.

\section{REMEDi}

The REMEDi system (Bio-Rad) is a multi-column based HPLC procedure with a full UV spectral scanning from 200 $\mathrm{nm}$ to $300 \mathrm{~nm}$. It uses two polymeric cartridges for on-line sample preparation and two analytical cartridges for separation. One analytical cartridge contains unmodified silica that separates basic drugs. Acidic drugs were removed by the second cleaning cartridge. Serum samples were prepared by mixing $150 \mu \mathrm{L}$ of an internal standard solution with $750 \mu$ Lserum. The internal standard solution consists of $N$-ethylnordiazepam and chlorpheniramine, as previously described $(3,4)$. The mixture was filtered through a $0.2 \mu \mathrm{m}$ filter unit, and the filtrate was centrifuged before injection. The injection volume was $0.5 \mathrm{~mL}$. The computer software (version 4.11), indicates it has a identification library of 555 drugs and metabolites.

The REMEDi procedure detects alprazolam, bromazepam, 
brotizolam, clonazepam, diazepam, estazolam, flunitrazepam, flurazepam, lorazepam, medazepam, midazolam, nitrazepam, oxazepam, prazepam, temazepam, and triazolam. The procedure, on occasions, detects a related compound but fails to positively identify that compound. In this situation, the REMEDi software will provide a "candidate list" which lists all possible candidates for that compound. The lower limits of detection (LOD) for benzodiazepines by REMEDi HS (a upgrade version of REMEDi) were: alprazolam. 0.25; bromazepam, 0.4; chlordiazepoxide, 0.35 ; clonazepam, 0.25; diazepam, 0.25; estazolam: 0.25; flunitrazepam, 0.8; flurazepam, 0.1; lorazepam, 0.4; nitrazepam, 1.0; midazolam, 0.15 (all in $\mu \mathrm{g} / \mathrm{mL}$ ).

\section{RESULTS}

\section{Immunoassay (aca Analyzer) for Benzodiazepines}

Among the 133 serum samples, 127 (approximates 95\%) were found positive for benzodiazepines, and 6 negative. Among these 127 aca positive samples, 13 were found negative by HPLC (data not show) and all 6 aca negative samples contained HPLC detectable benzodiazepines (Table 1). These findings indicate aca has high sensitivity but relatively low specificity as compared with HPLC procedure.

\section{Benzodiazepines Detection By HPLC}

HPLC detected the presence of benzodiazepines in 120 of the 133 samples analyzed (90\%). Among the 127 positive samples by aca analyzer, 114 samples were positive by HPLC. The remainder 13 cases were considered to be false positives by aca analyzer. Among the 6 negative samples by aca analyzer, 4 were found to contain bromazepam and 2 to contain flunitrazepam by HPLC. These 6 negative cases were therefore considered to be false negatives by aca analyzer (Table 1). Furthermore, 12 different compounds were qualitatively and quantitatively detectable by HPLC whereas aca analyzer provided only the presence of immunoactivity of benzodiazepines as a whole.

\section{REMEDi Detection of Benzodiazepines}

The REMEDi procedure detected a total of 84 benzodiazepine positives out of the 133 samples (approximates 63\%). Among the 127 positive samples by aca analyzer; 80 of them were positive by REMEDi procedure. Among the 6 negative samples by aca analyzer, 4 were found to contain bromazepam by REMEDi. REMEDi was able to qualitatively detect 11 of the 12 individual benzodiazepines except clonazepam, which could only be determined by HPLC and aca analyzer (Table 1). From our data, REMEDi procedure was excellent in the detection of bromazepam, flurazepam, lorazepam, triazolam and, to a much lesser degree, flunitrazepam and nitrazepam. The specificity of the benzodiazepine class compounds iden-
TABLE 1. Comparison of Sensitivity and Specificity in the Determination of Benzodiazepines in 133 Clinical Samples by aca Analyzer, REMEDi, and HPLC Procedures ${ }^{\mathrm{a}}$ When Lower Determination Threshold Set as $0.3 \mu \mathrm{g} / \mathrm{ml}$

\begin{tabular}{lccc}
\hline Compounds & $\begin{array}{c}\text { No. of samples } \\
\text { positive by } \\
\text { HPLC }\end{array}$ & $\begin{array}{c}\text { No. of samples } \\
\text { positive by } \\
\text { REMEDi }\end{array}$ & $\begin{array}{c}\text { No. of samples } \\
\text { positive by } \\
\text { aca }\end{array}$ \\
\hline Alprazolam & 1 & 1 & 1 \\
Bromazepam & 8 & $7[1]$ & $4\{4\}$ \\
Chlordiazepoxide & 5 & 4 & 5 \\
Clonazepam & 1 & 0 & 1 \\
Diazepam & 39 & $32[4]$ & 39 \\
Estazolam & 14 & $12[1]$ & 14 \\
Flunitrazepam & 17 & $6[4]$ & $15(2)$ \\
Flurazepam & 11 & 11 & 11 \\
Lorazepam & 3 & 3 & 3 \\
Nitrazepam & 15 & $3[5]$ & 15 \\
Triazolam & 4 & 4 & 4 \\
Midazolam & 2 & $1[1]$ & 2 \\
Total: & 120 & 84 & 114 \\
\hline
\end{tabular}

${ }^{\text {a }}$ The aca procedure detected a total of 127 positive cases (95\%) with 13 cases not confirmed by HPLC or REMEDi procedures and thus considered false positive. False aca positive data not shown. \{ \}, Number of samples positive by REMEDi and HPLC with corresponding negative aca analyzer; ( ), Number of samples positive by HPLC with corresponding negative aca analyzer; [ ], Number of samples with compounds detected but listed as candidates by REMEDi which were positively identified by aca analyzer.

tified by REMEDi were in total agreement with HPLC results (Fig. 1); that is, there were no false positives. Furthermore, in our sample series, the REMEDi procedure showed good agreement with the HPLC method in the detection of flurazepam (11 of 11 samples), lorazepam (3 of 3 samples), triazolam (4 of 4), and bromazepam (7 of 8) but displayed poor sensitivity for flunitrazepam (6 of 17) and particularly nitrazepam (3 of 15) as compared to HPLC.

Forty-nine (49) cases of the 133 samples were found to have low benzodiazepines levels $(0.3-1.0 \mu \mathrm{g} / \mathrm{mL})$. The presence of benzodiazepines were subsequently confirmed by HPLC. (Table 1). Among these 49 cases, the presence of benzodiazepines was detectable in 22 samples (44.9\%) by REMEDi procedure. Within the group of aca-positive samples, 16 were reported by REMEDi method as in the "candidate list" but the individual compounds were not positively identified (Table 1). When samples with benzodiazepines concentrations about $1.0 \mu \mathrm{g} / \mathrm{mL}$, the REMEDi findings were in total agreement with HPLC results, except one sample that contained both nitrazepam and flunitrazepam and was reported by REMEDi method as in the "candidate list " (Table 1 and Table 2).

Additionally, REMEDi procedure can detect other some nonbenzodiazepine drugs in the same sample, a possible advantage in clinical setting as previously observed by Chen et al (5). Within this group of 120 benzodiazepine positive patients (by HPLC), the serum samples of 20 


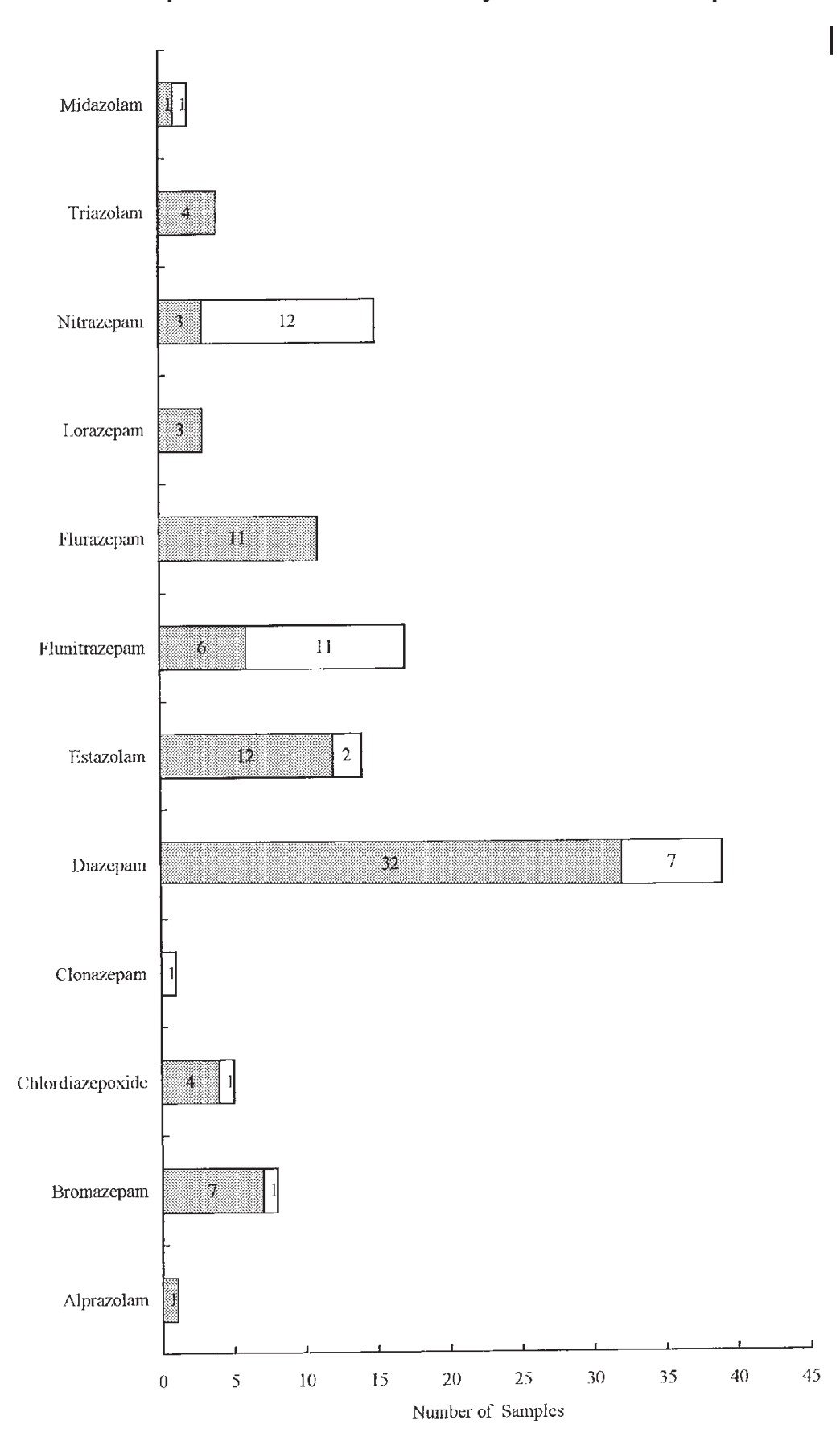

Fig. 1. Comparison of the sensitivity in the detection of benzodiazepines in clinical serum samples by REMEDi and conventional HPLC procedures. Shaded bar, number of samples positive by REMEDi pro- cedure; clear bar plus shaded bar, number of samples positive by conventional HPLC procedure.

\section{DISCUSSION}

We comparatively evaluated the performance of aca analyzer, REMEDi, and conventional HPLC in the detection of 12 different benzodiazepines in 133 serum samples. The objective of this study was to test whether REMEDi procedure could replace aca analyzer as a rapid and reliable clinical emergency drug screening method. 
TABLE 2. Comparison of REMEDi, aca Analyzer, and HPLC Analysis in Samples With Benzodiazepines Concentration Ranges Between 0.3-1.0 $\mu \mathrm{g} / \mathrm{ml}^{\mathrm{a}}$

\begin{tabular}{lllc}
\hline Compounds & $\begin{array}{c}\text { REMEDi } \\
\text { positive }\end{array}$ & $\begin{array}{c}\text { aca } \\
\text { positive }\end{array}$ & $\begin{array}{c}\text { HPLC } \\
\text { positive }\end{array}$ \\
\hline Bromazepam & $7[1]$ & $4\{4\}$ & 8 \\
Chlordiazepoxide & 2 & 3 & 3 \\
Clonazepam & 0 & 1 & 1 \\
Diazepam & $3[4]$ & 11 & 11 \\
Estazolam & $1[1]$ & 3 & 3 \\
Flunitrazepam & $1[3$ & $9(2)$ & 11 \\
Flurazepam & 1 & 1 & 1 \\
Lorazepam & 2 & 2 & 2 \\
Nitrazepam & $3[5]$ & 14 & 14 \\
Triazolam & 2 & 2 & 2 \\
Midazolam & $0[1]$ & 1 & 1 \\
\hline
\end{tabular}

${ }^{a}\{\}$, Number of samples positive by REMEDi and HPLC but negative by aca analyzer; ( ), Number of samples positive by HPLC but negative by aca analyzer; [ ], Number of samples with compounds detected as candidates by REMEDi but reported as positive by aca analyzer.

From data presented in Table 1, alprazolam, lorazepam, and triazolam all can be identified by REMEDi. The sensitivity of benzodiazepine is about $0.5 \mu \mathrm{g} / \mathrm{mL}$ with considerable variation among individual compound; although from manufacturer data, alprazolam can be detected at $0.1 \mu \mathrm{g} / \mathrm{mL}$ level. In this group of samples tested, the standard immunoassay (aca analyzer) apparently failed to detect bromazepam and estazolam in selected samples while these compounds were easily detectable by REMEDi. Flurazepam is highly detectable by REMEDi because it is very basic and has basic metabolites. REMEDi sensitivity for bromazepam, estazolam, flurazepam, lorazepam, triazolam, and midazolam apparently is high, since uniform positive detection can be achieved even when concentration of the compound is below $1.0 \mu \mathrm{g} / \mathrm{mL}$ but above the $0.3 \mu \mathrm{g} / \mathrm{mL}$ level.

Demoxepam is a metabolite of chlordiazepoxide. During this study we found two samples contained REMEDi-detectable demoxepam (data not shown). In our series, we found that when the diazepam (the most commonly used drug in southern Taiwan) level in the test sample was below the sensitivity range of REMEDi $(0.32-0.45 \mu \mathrm{g} / \mathrm{mL})$, the presence of diazepam was identifiable only by HPLC. Although flunitrazepam is listed as a detectable candidate by REMEDi, we found that when the concentration was below $0.29 \mu \mathrm{g} /$ $\mathrm{mL}$, the compound was not identifiable by REMEDi, but detectable by HPLC. The detection of nitrazepam by REMEDi required a concentration above $0.51 \mu \mathrm{g} / \mathrm{mL}$ for positive identification. This relative insensitivity by REMEDi for both nitrazepam and flunitrazepam probably is due to their acidic nature. Our data on nitrazepam and flunitrazepam are similar to that reported by Sadeg et al.(10). Therefore, when samples with these benzodiazepines concentration in the ranges of $0.3-1.0 \mu \mathrm{g} / \mathrm{mL}$, detection sensitivity by REMEDi became varied. Furthermore, since nitrazepam is one of the most clinically important benzodiazepines for life threatening intoxication, the relative insensitivity by REMEDi on this compound can be considered as a weakness of this procedure for clinical emergency situation, although higher level of nitrazepam was apparently detectable by REMEDi.

The majority of the 36 HPLC-positive but REMEDi-negative samples had an immunoassay benzodiazepine concentration reading close to the cut-off of $0.3 \mu \mathrm{g} / \mathrm{mL}$, hence they were well below the sensitivity ranges of REMEDi. These data further illustrate that detection sensitivity is a limiting factor for REMEDi system for clinical samples with low benzodiazepine concentrations.

Rapid turn-around time is a major consideration in emergency toxicology screening. REMEDi analysis requires about 30 min to complete while conventional HPLC requires about $2 \mathrm{hr}$, mainly due to tedious off-line sample preparation. However, for detection of benzodiazepines, the sensitivity of the REMEDi system is inferior to standard conventional HPLC (Fig. 1) and for some compounds, aca analyzer. Sensitivity of REMEDi system probably can be increased by off-line preconcentration of samples using solid-phase or solvent extraction, but this would be time consuming and may introduce unwanted complexity.

From data shown in Figure 1, diazepam was the most commonly detected benzodiazepine whereas alprazolam was the least detected compound in samples obtained from patients in southern Taiwan. Furthermore, most of the diazepam positive samples had a concentration higher than $1.0 \mu \mathrm{g} / \mathrm{mL}$. Other nonbenzodiazepine drugs most frequently detected in the same samples by REMEDi were caffeine, followed by diphenhydramine and cimetidine.

Our data suggest that REMEDi system is a useful screening method for emergency preliminary qualitative determination of clinically significant or toxic levels of benzodiazepines and their metabolites in serum samples. Advantages of the REMEDi profiling system are simplicity, speed, and good specificity. Although REMEDi is much less effective or less sensitive than the aca assay or HPLC procedure for the detection of benzodiazepines in serum, the procedure displayed high specificity equal to HPLC. The main weakness of the system is its overall sensitivity which is apparently inferior to HPLC and for some compounds, aca. In emergency situations, however, results from REMEDi should provide critical information to physicians for initiating necessary treatment in patients with high or toxic level of benzodiazepines while samples with therapeutic or low level of benzodiazepine should be analyzed by conventional HPLC procedure.

\section{ACKNOWLEDGMENT}

This work was not financially supported by instrument or reagent manufacturers. 


\section{REFERENCES}

1. Puopolo PR, Pothier ME, Volpiicelli SA, Flood JG. Single procedure for detection, confirmation and quantitation of benzodiazepines in serum by liquid chromatography with photodiode-array detection. Clin Chem 1991;37:701-706.

2. Hansen JB, Lau HP, Janes CJ, Miller WK, Wiebe DA. Rapid and specific cyclosporine assay for the Du Pont aca discrete clinical analyzer performed directly on whole blood. J Clin Lab Anal 1991;5:187-192.

3. Adams AK, Essien H, Binder SR. Identification of drugs in physiological fluids following on-line liquid chromatographic purification and analysis. Annales de Biologie Clinique 1991;49:291-297.

4. Binder SR, Regalia M, McEachern B, Mazhar M. Automated liquid chromatographic analysis of drugs in urine by on-line sample clean up and isocratic multi-column separation. J Chromatogr 1989;473:325-341.

5. Chen JS, Chang KJ, Chang RC, Lai SJ, Binder SR, Essien H. The development of a broad-spectrum toxicology screening program in Taiwan. Clin Toxicol 1995;33:581-589.
6. Young NC, Tsai KS, Lai SJ. The use of a broad-spectrum drug identification system in emergency clinical toxicology screening. Paper presented at $25^{\text {th }}$ annual Society of Forensic Toxicology Meeting, Baltimore, MD, USA; 1995.

7. Poklis A, Edinboro LE. Remedi drug profiling system readily distinguishes between cyclobenzaprine and amitriptyline in emergency toxicology urine specimens. Clin Chem 1992;38:2349-2350.

8. Demedts P, Wauters A, Franck F, Neels H. Evaluation of the Remedi Drug Profiling System. Eur J Clin Chem Clin Biochem 1994;32:409-417.

9. Lai SJ, Binder SR, Essien H, Wen KC. Identification of western medicines as adulterants on Chinese herbal medicines using a broadspectrum drug screening HPLC system. J Chromatogr 1995;18: 2861-2875.

10. Sadeg N, Francois G, Petit B, Dutertre-Catella H, Dumontet M. Automated liquid chromatographic analyzer used for toxicology screening in a general hospital: 12 months' experience. Clin Chem 1997;43:498-504. 